\title{
Circulating Micro RNA- 21 and - 92a as Biomarkers of Colorectal Cancer
}

\author{
Ebada Said ${ }^{1}$, Ahmed Zidan², Ahmed Nawar ${ }^{2}$, Naglaa Alhusseini, \\ Elwy Soliman ${ }^{4}$, Rasha Shaker ${ }^{5}$, Waleed Naguib6, Kamal Rady \\ and Hosam Baiumy ${ }^{1}$. \\ Hepatology, Gastroenterology and Infectious Diseases ${ }^{1}$, Benha University, General Surgery \\ and Oncosurgery ${ }^{2}$, Benha University, Medical Biochemistery and Molecular Biology, \\ Benha University, Internal Medicine ${ }^{4}$, Minia University, Community Medicine ${ }^{5}$, Benha University, \\ Internal Medicine ${ }^{6}$, El-Senbellawain General Hospital and Anatomy ${ }^{7}$, Al Azhar University.
}

Corresponding Author Ebada Mohamed Said

Mobile:

$+201220115116$

E mail: ebadamsaid@yahoo. com

Key words: Colorectal cancer (CRC); miRNA-21; miRNA-92a; biomarkers
Background and study aim: Colorectal cancer (CRC) is a major cause of cancerrelated deaths in both men and women. Colonoscopy is the most reliable tool for CRC diagnosis, but its complexity and costs hamper its wide application. There is a pressing need for new non-invasive biomarkers to improve early diagnosis of CRC. Aim was to assess serum micro RNA (miR) -21and miR-92a for diagnosis of CRC.

Patients and Methods: This comparative cross sectional study was carried out on 50 subjects. The cases group comprised 35 consecutive treatment naive patients with sporadic CRC proved by colonoscopy and histopathology of the biopsied specimens as well as abdominal CT which all together helped in tumor staging applying TNM, Duke's and MAC Coller stages. Serum carcinoembryonic antigen (CEA) and cancer antigen (CA) 19-9 were also assessed. Fifteen matched healthy subjects (with normal colonoscopy) served as the control group.

\section{INTRODUCTION}

Colorectal cancer (CRC) is the third most common cancer diagnosed in both men and women [1]. The lifetime risk of developing CRC is about 1 in $21(4.7 \%)$ for men and 1 in $23(4.4 \%)$ for women. Many factors increase the risk including: obesity, diet high in red meat (such as beef, pork, lamb, or liver) and processed meat, smoking, heavy alcohol use, history of inflammatory bowel disease, family history of CRC or adenomatous polyps and diabetes mellitus [1].
Results: Serum levels of miR-21 and miR-92a were significantly higher $(\mathrm{P}<0.001)$ in cases compared to the control $(5.53 \pm$ $0.17 \neq 4.82 \pm 0.20$ and $7.01 \pm 0.234 \neq 6.56$ $\pm 0.20 \log \mathrm{RU}$, respectively). Serum miR21 and miR-92a levels revealed a significant positive relation with tumor size and TNM and MAC Coller stages $(\mathrm{P}<0.05)$. No significant relationship was detected between either serum miR-21 or miR-92a levels with age or sex. Applying ROC curve, at a cutoff value of $\geq 5.25 \log \mathrm{RU}$, serum miR-21 was $94.3 \%$ sensitive and 93.3\% specific for detection of CRC with an $\mathrm{AUC}=0.99$ and serum miR-92a level at a cut off value $\geq 6.75 \log \mathrm{RU}$, was 91.4\% sensitive and $80 \%$ specific for detection of CRC with $\mathrm{AUC}=0.91$. When both markers were combined, the sensitivity and specificity were $97.1 \%$ and $93.3 \%$ respectively.

Conclusion: Serum miR-21 and miR-92a levels represent a sensitive and specific tool for CRC diagnosis, with higher accuracy of miR-21.
The CRC mortality rates can be decreased by early diagnosis through screening. However, the present CRC screening techniques [colonoscopy, faecal occult blood test (FOBT), and carcinoembryonic antigen (CEA) test] are limited by their difficulties and costs beside uncertain or delayed results [2].

Although colonoscopic screening for $\mathrm{CRC}$ is the most reliable tool; its difficulties and costs have hampered its wide application. On the other hand, FOBT has the limitation 
of low sensitivity and dietary restrictions [3]. Thus, there is a pressing need for new noninvasive biomarkers to improve the early detection of CRC [4].

The discovery of micro RNAs (miRNAs), that play important roles in oncogenesis, has opened new opportunities of non-invasive tests for the early diagnosis of cancers [5].

MiRNAs are a family of small, noncoding RNAs (19-22 nucleotides) which post-transcriptionally regulate gene expression. In general, miRNAs are transcribed as a group called the pri-miRNA complex, which is cleaved in the nucleus to form the pre-miRNA which is then translocated to the cytoplasm where they undergo final maturation into a functional miRNA [6]. Studies have shown that profiles of miRNA expression differ between normal- and tumour- tissues and vary among different tumour types [7]. Aberrant miRNA expression profiles have been identified and emerged as potential screening biomarkers for CRC $[8,9]$.

Although most previous studies on miRNA expression have been performed on tissue specimens, some studies have shown diagnostic and prognostic potential for circulating miRNAs because tumor-derived miRNAs can be present in blood and appear to be stably protected from endogenous ribonuclease activity in the circulation [10].

MiR-21 is an oncogenic miRNA that modulates the expression of multiple cancer-related target genes such as PTEN, TPM1, and PDCD and has been shown to be overexpressed in various human tumors [11]. In addition, miR-21 expression is upregulated in CRC tissues, is elevated during tumor progression, and is also associated with poor survival and response to chemotherapy [12]. Significantly elevated plasma miR-21 expression in CRC was concluded in many studies $[\mathbf{1 1}, \mathbf{1 3}]$. On the other hand, MiR92a is part of the miR-17-92 gene cluster located at chromosome 13q13. As a known oncomir, the miR-17-92 cluster can promote cell proliferation, suppress apoptosis of cancer cells, induce tumor angiogenesis and accelerate tumor progression. Elevated expression of miR-92a has been observed in CRC, lung and thyroid cancers suggesting an important role in tumorigenesis. Many studies reported that circulating miR-92 is a potential biomarker for CRC diagnosis [13,14]. The aim of the present study was to assess miR-
21 and $\mathrm{miR}-92 \mathrm{a}$ expression levels as a stable blood-based biomarker for detection of CRC.

\section{PATIENTS AND METHODS}

This comparative cross sectional study was carried out on 50 subjects. The cases group comprised 35 consecutive patients with CRC (19 males and 16 females) who were attending the Departments of Hepatology, Gastroenterology and Infectious Diseases and General Surgery at Benha University Hospitals, within the period between January 2015 and October 2016. All had sporadic CRC, proved by colonoscopy and histopathology. Fifteen age and sex matched apparently healthy subjects who had normal routine laboratory investigations and colonoscopy served as healthy control. The indication for colonoscopy was unexplained abdominal pain and/or altered bowel habits. Patients with familial adenomatous polyposis or hereditary non-polyposis CRC, those who received chemotherapy or radiotherapy and pregnant female patients were excluded from the study. The study protocol was approved by the Ethics Committee of Benha Faculty of Medicine, Benha University. An informed written consent was obtained from the participants.

\section{All the studied 35 cases were subjected to the following :}

Full history taking focusing on: family history of CRC, recent- onset constipation, bleeding per rectum, significant weight loss, and/or anemia of unexplained aetiology. Thorough clinical examination including general examination focusing on: cachexia, pallor and lymphadenopathy. Local abdominal examination focusing on: palpable abdominal masses, ascites, LN and PR examination.

Laboratory investigations, including: stool analysis, complete blood count (CBC), erythrocyte sedimentation rate (ESR), random blood sugar (RBS), liver profile tests, including: alanine aminotransferase (ALT), aspartate aminotransferase (AST), alkaline phosphatase (ALP), serum albumin, serum bilirubin (total and direct) and prothrombin time (PT), serum creatinine and blood urea and Serum CEA and CA19-9.

Detection of miRNA- 21 expression level using real-time PCR technique: Extraction of total RNA including microRNA-21 from plasma samples using microRNA extraction kit according to the 
manufacturer's instructions [15]. Relative quantitation of microRNA-21 level using realtime quantitative PCR (RT-PCR) according to the manufacturer's instructions [16].

Detection of miRNA- 92a expression level using real-time PCR technique: Extraction of total RNA including microRNA-92a from plasma samples using miRNA extraction kit according to [15]. Relative quantitation of microRNA-92a level using real-time quantitative PCR (RT-PCR) according to Siege et al. [17].

Pelvi-abdominal ultrasonography and computed tomography (CT scan) were done. Complete colonoscopy was done under sedation with patient preparation through Low-volume Polyethylene glycol-based lavage solution with ascorbic acid (Low-volume PEG-ELS with ascorbic acid) administered as split-dose regimen. Multiple biopsies were taken from any suspected lesion, and were sent for histopathological examination. TNM, Duke's and MAC Coller staging systems were applied.

\section{Statistical analysis [18] :}

The collected data were tabulated and analyzed using SPSS version 16 soft ware (SpssInc, Chicago, ILL Company). Categorical data were presented as number and percentages while quantitative data were expressed as mean \pm standard deviation, median and range. Chi square test (X2) or Fisher's exact test (FET) were used to analyze categorical variables. Quantitative data were tested for normality using ShapiroWilks test, assuming normality at $\mathrm{P}>0.05$. Student " $t$ " test was used for normally distributed variables, while Man Whitney U (MWU) test, Kruskal Wallis test and Spearman's correlation coefficient (rho) were used for not normally distributed data. ROC curve was used to determine cutoff values of miRNA-21 and miR-92a with optimum sensitivity and specificity in prediction of patients with CRC. $\mathrm{P}$ value $\leq 0.05$ was considered significant.

\section{RESULTS}

The studied cases included 19 males (56\%) and 16 females $(44 \%)$ with a mean age of $50.6 \pm$ 15.1 ys. Age categories among the studied cases showed $26 \%$ below 40 years and $63 \%$ above 50 years.

The main presenting symptom in the studied cases was bleeding per rectum $(15 / 35=42.9 \%)$ followed by recent onset constipation $(13 / 35=$ $37.1 \%)$ and significant weight loss $(7 / 35=20 \%)$. Positive family history was found in $(6 / 35=17 \%)$ of the studied cases.

Colonoscopic examination of the studied cases revealed that the main lesion site of CRC was colon $(24 / 35=68.6 \%)$, followed by rectum $(9 / 35$ $=25.7 \%)$ and lastly rectosigmoid $(2 / 35=5.7 \%)$. Small lesions $(<2.5 \mathrm{~cm})$ were seen in 4 cases $(11.4 \%)$, medium sized ones $(2-5 \mathrm{~cm})$ were $37.1 \%$ (13 cases), while large lesions were seen in 18 cases $(51.4 \%)$. Mass was the main lesion seen $(94.2 \%)$ with only one case showed ulcer and another one showed a stricture. The main histo-pathological CRC types in the studied cases were adenocarcinoma $(24 / 35=68.6 \%)$ followed by mucinous adenocarcinoma $(9 / 35=25.7 \%)$ and signet ring cell adenocarcinoma $(2 / 35=$ $5.7 \%)$.

The majority of the studied cases were Dukes B (88.6\%). According to TNM staging, cases with TIIB were $(54.3 \%)$ followed by TIIA $(34.3 \%)$. Three cases only were Tis $(8.5 \%)$. None of the studied cases had nodal involvement or metastases (all were NOM0).

Table (1): Circulating miR-21 and miR-92a levels in the studied groups.

\begin{tabular}{|c|c|c|c|c|c|c|}
\hline \multirow[t]{2}{*}{ Variable } & \multicolumn{2}{|c|}{$\begin{array}{c}\text { Cases } \\
\left(N^{\circ}=35\right)\end{array}$} & \multicolumn{2}{|c|}{$\begin{array}{l}\text { Controls } \\
\left(\mathrm{N}^{\circ}=15\right)\end{array}$} & \multirow{2}{*}{$\begin{array}{c}\text { Z of } \\
\text { MWU } \\
\text { test }\end{array}$} & \multirow[t]{2}{*}{$\mathbf{P}$} \\
\hline & Mean \pm SD & Range & Mean \pm SD & Range & & \\
\hline MiR-21 level & $5.53 \pm 0.17$ & $4.93-5.67$ & $4.82 \pm 0.20$ & $4.62-5.41$ & 5.55 & $<0.001 * *$ \\
\hline MiR-92a level & $7.01 \pm 0.234$ & $6.46-7.33$ & $6.56 \pm 0.20$ & $6.05-6.9$ & 4.56 & $<0.001 * *$ \\
\hline
\end{tabular}

\footnotetext{
**Highly signifiant $\mathrm{P}$ value
} 
Table (2): Mean values of serum miR-21\& miR-92a in relation to colonoscopy findings.

\begin{tabular}{|c|c|c|c|c|c|c|c|c|c|c|c|c|}
\hline & \multirow{2}{*}{ Lesion } & \multirow{2}{*}{$\begin{array}{c}\mathrm{N}^{\circ} \\
(\mathrm{T}=35)\end{array}$} & \multicolumn{2}{|c|}{ Serum miR-21 $(\log \mathrm{RU})$} & \multirow{2}{*}{ KWT } & \multirow{2}{*}{$\mathbf{P}$} & \multirow{2}{*}{ Sig pairs } & \multicolumn{2}{|c|}{ microRNA-92 } & \multirow{2}{*}{ KWT } & \multirow{2}{*}{$P$} & \multirow{2}{*}{ Sig pairs } \\
\hline & & & Mean \pm SD & Range & & & & Mean \pm SD & Range & & & \\
\hline \multirow{3}{*}{ Site } & Colon & 24 & $5.51 \pm 0.19$ & $4.93-5.67$ & 0.28 & \multirow{3}{*}{$\begin{array}{l}\infty \\
\infty \\
0\end{array}$} & \multirow[t]{3}{*}{ 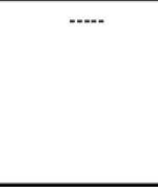 } & $6.98 \pm 0.25$ & $6.94-7.32$ & \multirow{3}{*}{4.02} & \multirow{3}{*}{$\frac{2}{0}$} & \multirow{3}{*}{$\cdots$} \\
\hline & Rectum & 9 & $5.56 \pm 0.09$ & $5.41-5.66$ & & & & $7.14 \pm 0.13$ & $6.94-7.32$ & & & \\
\hline & $\begin{array}{l}\text { Recto- } \\
\text { sigmoid } \\
\end{array}$ & 2 & $5.57 \pm 0.05$ & $5.54-5.61$ & & & & $6.82 \pm 0.02$ & $6.8-6.84$ & & & \\
\hline \multirow{3}{*}{ Size } & Small & 4 & $5.22 \pm 0.31$ & $4.93-5.54$ & \multirow[t]{3}{*}{14.5} & \multirow{3}{*}{ 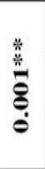 } & \multirow{3}{*}{$\begin{array}{l}\text { Small } \neq \text { medium } \\
\text { Small } \neq \text { large }\end{array}$} & $6.75 \pm 02$ & $6.46-6.94$ & \multirow[t]{3}{*}{11.9} & \multirow{3}{*}{ : } & \multirow{3}{*}{$\begin{array}{l}. \text { Small } \neq \text { medium } \\
\text { Medium } \neq \text { large }\end{array}$} \\
\hline & Medium & 13 & $5.51 \pm 0.09$ & $5.41-5.65$ & & & & $6.93 \pm 024$ & $6.48-7.28$ & & & \\
\hline & Large & 18 & $5.61 \pm 0.07$ & $5.44-5.67$ & & & & $7.13 \pm 015$ & 6.8-7.33 & & & \\
\hline \multirow{3}{*}{ 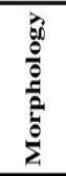 } & Mass & 33 & $5.54 \pm 0.14$ & $4.93-5.67$ & \multirow{3}{*}{2.93} & \multirow{3}{*}{ त̂? } & \multirow[t]{3}{*}{$\ldots+\cdots$} & $7.02 \pm 023$ & $6.46-7.33$ & & \multirow{3}{*}{$\stackrel{m}{3}$} & \multirow[t]{3}{*}{-...-.. } \\
\hline & Ulcer & 1 & $5.63 \pm \ldots-$ & $5.64-5.64$ & & & & $6.79 \pm--$ & $6.8-6.8$ & & & \\
\hline & Stricture & 1 & $4.98 \pm \ldots .-$ & $4.98-4.98$ & & & & $6.94 \pm--$ & 6.94-6.94 & 2.19 & & \\
\hline \multirow{3}{*}{ 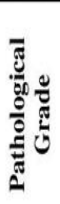 } & Grade I & 3 & $5.27 \pm 0.296$ & $4.93-5.45$ & \multirow{3}{*}{8.06} & \multirow{3}{*}{$\begin{array}{l}\stackrel{*}{*} \\
\stackrel{\infty}{0} \\
\\
\end{array}$} & \multirow[t]{3}{*}{$\mathrm{I} \neq \mathrm{III}$} & $5.21 \pm 0.286$ & $4.83-5.45$ & & \multirow{3}{*}{$\stackrel{*}{0}$} & \multirow[t]{3}{*}{ If IIII } \\
\hline & Grade II & 27 & $5.51 \pm 0.176$ & $4.98-5.65$ & & & & $5.41 \pm 0.186$ & $4.78-5.55$ & & & \\
\hline & Grade III & 5 & $5.64 \pm 0.028$ & $5.61-5.67$ & & & & $5.60 \pm 0.029$ & $5.51-4.67$ & 8.02 & & \\
\hline
\end{tabular}

*Significant $\mathrm{P}$ vaule

**Highly signifcicant $\mathrm{P}$ value

RU:relative uncertainty RU

A statistically significant relationship was found between serum- miR-21\& -miR-92a and the tumor size $(\mathrm{P}=0.001 \& 0.003$ respectively) as well as the tumor pathological grade $(\mathrm{P}=0.018 \& 0.017$ respectively), while tumour site and morphology showed non-significant relationships with serum miR-21 and miR-92a expression.

Table (3): Correlation between serum miR-21\& miR-92a and assessed variables in the cases group.

\begin{tabular}{|l|c|c|c|c|}
\hline \multirow{2}{*}{ Variable } & \multicolumn{2}{c|}{ miR-21 } & \multicolumn{2}{c|}{ miR-92a } \\
\cline { 2 - 5 } & rho & P & rho & P \\
\hline Age & 0.231 & $0.18(\mathrm{NS})$ & 0.076 & $0.66(\mathrm{NS})$ \\
\hline Hb\% & -0.138 & $0.43(\mathrm{NS})$ & 0.115 & $0.51(\mathrm{NS})$ \\
\hline RBCs & 0.083 & $0.063(\mathrm{NS})$ & 0.232 & $0.18(\mathrm{NS})$ \\
\hline WBCs & -0.041 & $0.81(\mathrm{NS})$ & -0.149 & $0.39(\mathrm{NS})$ \\
\hline PLTs & 0.477 & $\mathbf{0 . 0 0 8}(\mathbf{S})$ & -0.177 & $0.31(\mathrm{NS})$ \\
\hline RBS & 0.062 & $0.72(\mathrm{NS})$ & 0.234 & $0.17(\mathrm{NS})$ \\
\hline ALT & 0.058 & $0.74(\mathrm{NS})$ & 0.016 & $0.92(\mathrm{NS})$ \\
\hline AST & 0.055 & $0.75(\mathrm{NS})$ & 0.162 & $0.35(\mathrm{NS})$ \\
\hline ALP & -0.158 & $0.36(\mathrm{NS})$ & 0.199 & $0.25(\mathrm{NS})$ \\
\hline S.albumin & -0.1 & $0.57(\mathrm{NS})$ & -0.161 & $0.35(\mathrm{NS})$ \\
\hline T. Bilirubin & 0.126 & $0.47(\mathrm{NS})$ & 0.292 & $0.09(\mathrm{NS})$ \\
\hline Creat. & 0.246 & $0.15(\mathrm{NS})$ & 0.249 & $0.15(\mathrm{NS})$ \\
\hline BUN & 0.023 & $0.89(\mathrm{NS})$ & 0.052 & $0.77(\mathrm{NS})$ \\
\hline CEA & 0.131 & $0.45(\mathrm{NS})$ & 0.022 & $0.9(\mathrm{NS})$ \\
\hline CA19-9 & 0.161 & $0.35(\mathrm{NS})$ & 0.034 & $0.84(\mathrm{NS})$ \\
\hline
\end{tabular}

There was a statistically significant positive correlation between serum miR-21and platelets count $(\mathrm{P}=0.008)$, while there were non- significant correlations between serum miR-21 and miR-92a and the other assessed variables. 
Table (4) : Sensitivity and specifity of miRNA-21 and microRNA-92a for CRC diagnosis.

\begin{tabular}{|c|c|c|c|c|c|c|c|}
\hline Variable & Sens \% & Spec \% & PPV\% & NPV\% & AUC & 95\% CI & P \\
\hline miR -21 $\geq \mathbf{5 . 2 5} \log \boldsymbol{R U}$ & $94.3 \%$ & $93.3 \%$ & $97 \%$ & $87.5 \%$ & 0.99 & $0.97-1.0$ & $<\mathbf{0 . 0 0 1} * *$ \\
\hline miR -92a $\geq \mathbf{6 . 7 5} \log \boldsymbol{R U}$ & $91.4 \%$ & $80 \%$ & $91.4 \%$ & $80 \%$ & 0.91 & $0.83-0.99$ & $<\mathbf{0 . 0 0 1} * *$ \\
\hline Combined miR -21 \& miR -92a & $97.1 \%$ & $93.3 \%$ & $97.1 \%$ & $93.3 \%$ & & & \\
\hline
\end{tabular}

**Highly signifcicant $\mathrm{P}$ value

At a cut off value $\geq 5.25 \log \mathrm{RU}$, serum miR-21 was $94.3 \%$ sensitive and $93.3 \%$ specific for detection of $\mathrm{CRC}$, with $\mathrm{AUC}=0.99$. At a cut off value $\geq 6.75 \log \mathrm{RU}$, serum miR-92a was $91.4 \%$ sensitive and $80 \%$ specific for detection of CRC with $\mathrm{AUC}=0.91$. When both markers were combined, the sensitivity increased to $97.1 \%$ and specifity was $93.3 \%$.

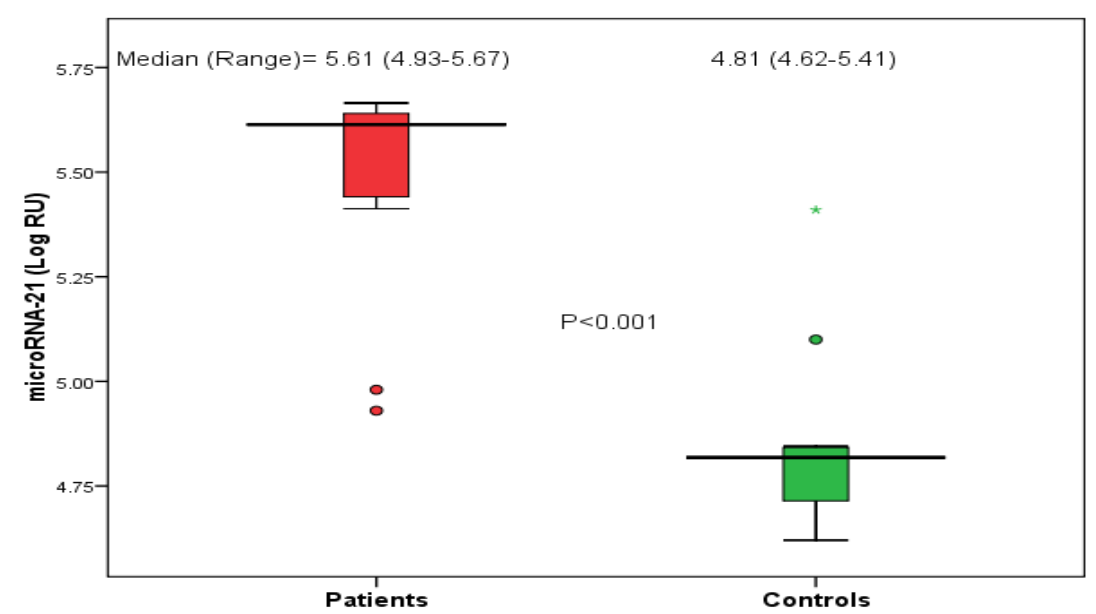

Fig. (1): Box plot showing the median and range of serum miR-21 among cases and control group

Serum miR-21 expression was significantly higher in the cases group compared to the control $(5.53 \pm$ $0.17 \neq 4.82 \pm 0.20 \log \mathrm{RU})$ with $(\mathrm{P}<0.001)$.

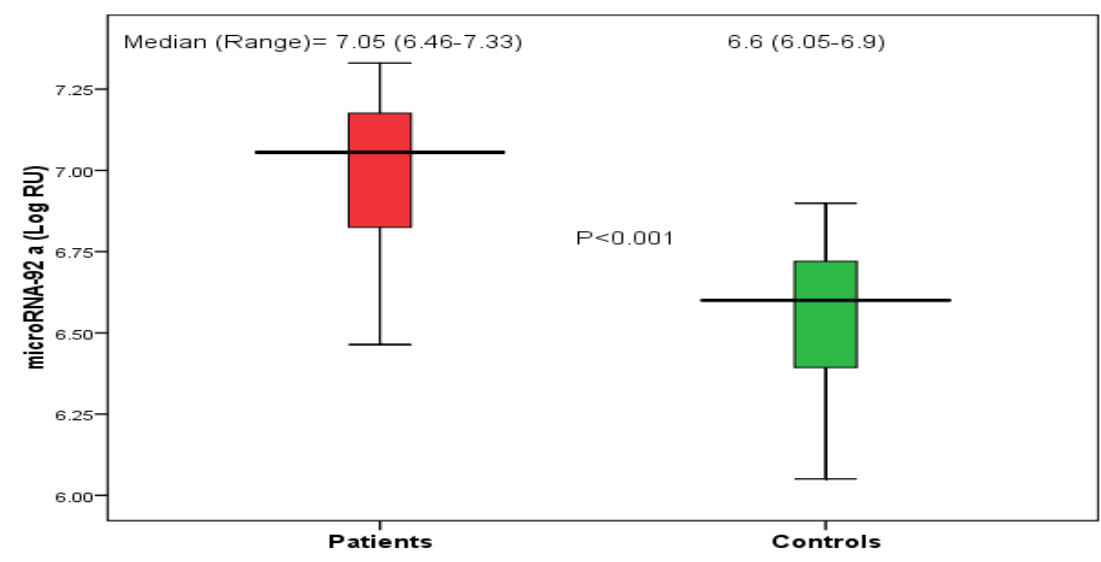

Fig. (2): Box plot showing the median and range of serum miR-92a in cases and control groups

Serum miR-92a expression was significantly higher in the cases group compared to the control $(7.01 \pm$ $0.234 \neq 6.56 \pm 0.20 \log \mathrm{RU})$ with $(\mathrm{P}<0.001)$. 


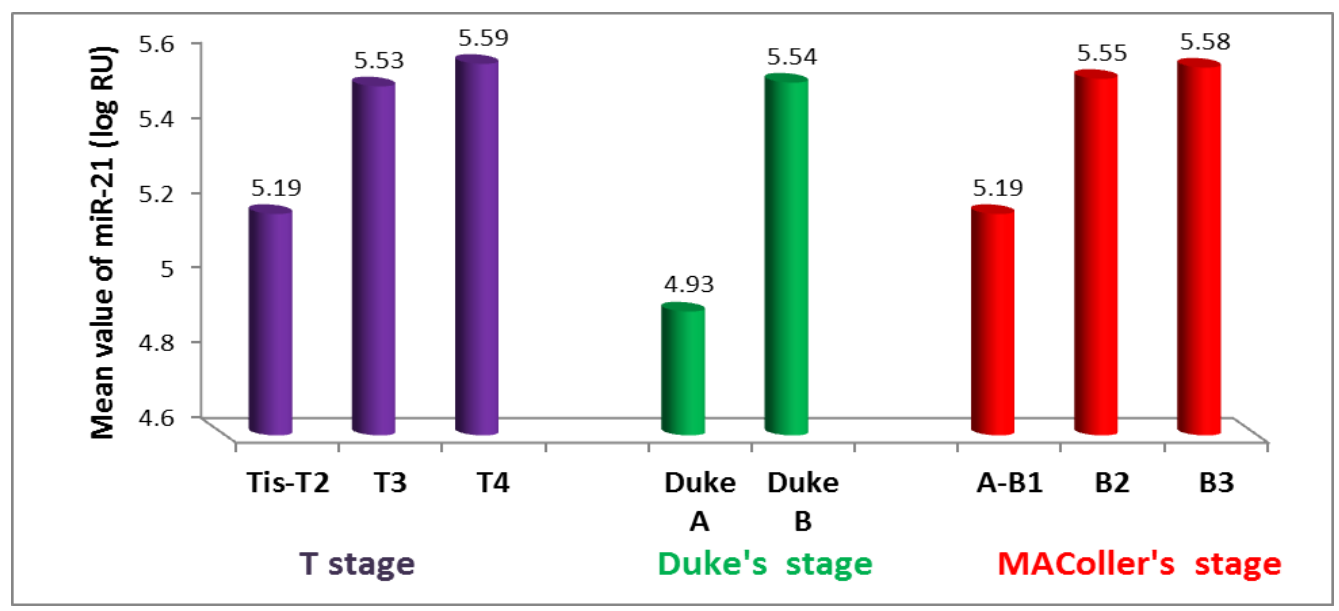

Fig. (3): Mean values of serum miR-21 in different CRC stages

A statistically significant positive relationship was found between $\mathrm{T}$ satge $(\mathrm{P}=0.01)$ as well as MAC Coller stage $(\mathrm{P}=0.008)$ and serum miR-21 expression, while a statistically non-significant relationship was found between Duke's Stage and serum miR- 21 expression.

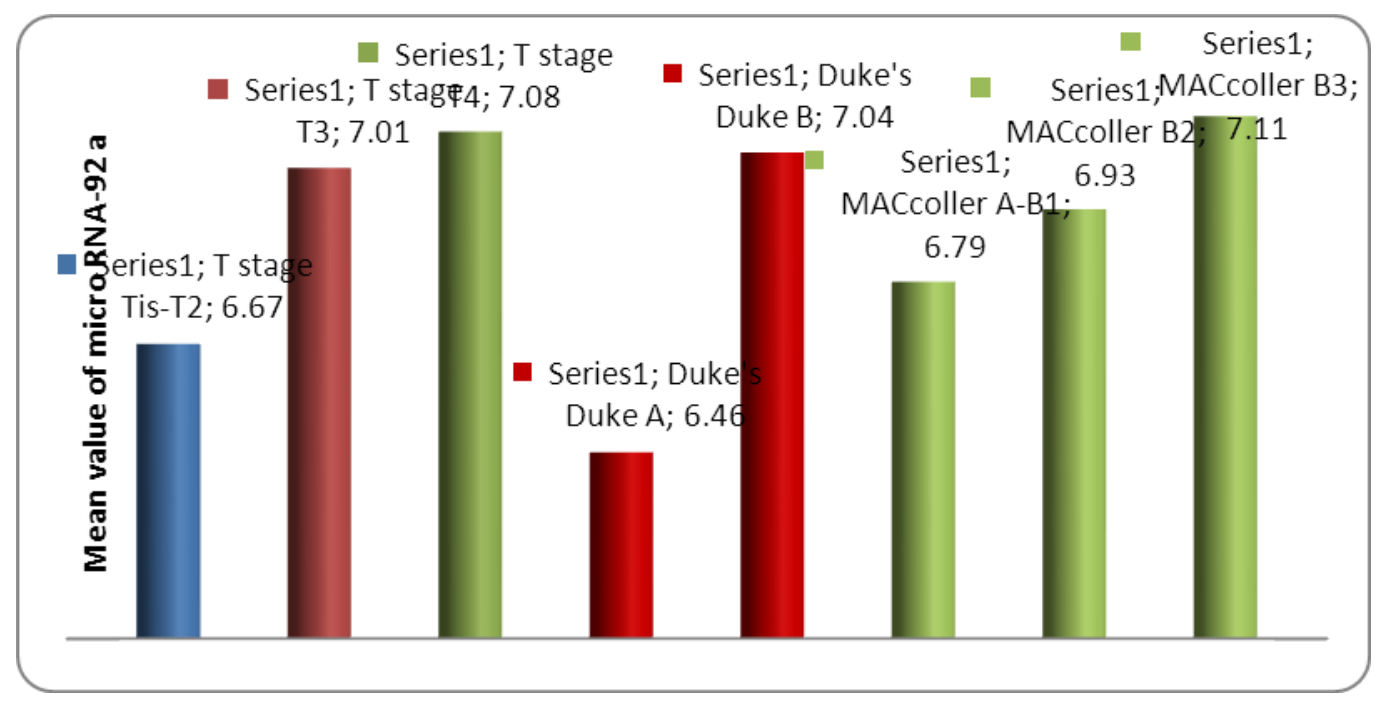

Fig. (4): Mean values of serum miR-92a in different CRC stages

A statistically significant positive relationship was found between $\mathrm{T}$ satge $(\mathrm{P}=0.03)$ as well as MAC Coller stage $(\mathrm{P}=0.031)$ and serum miR-92a expression, while a statistically non-significant relation was found between Duke's Stage and serum miR- 92a expression. 


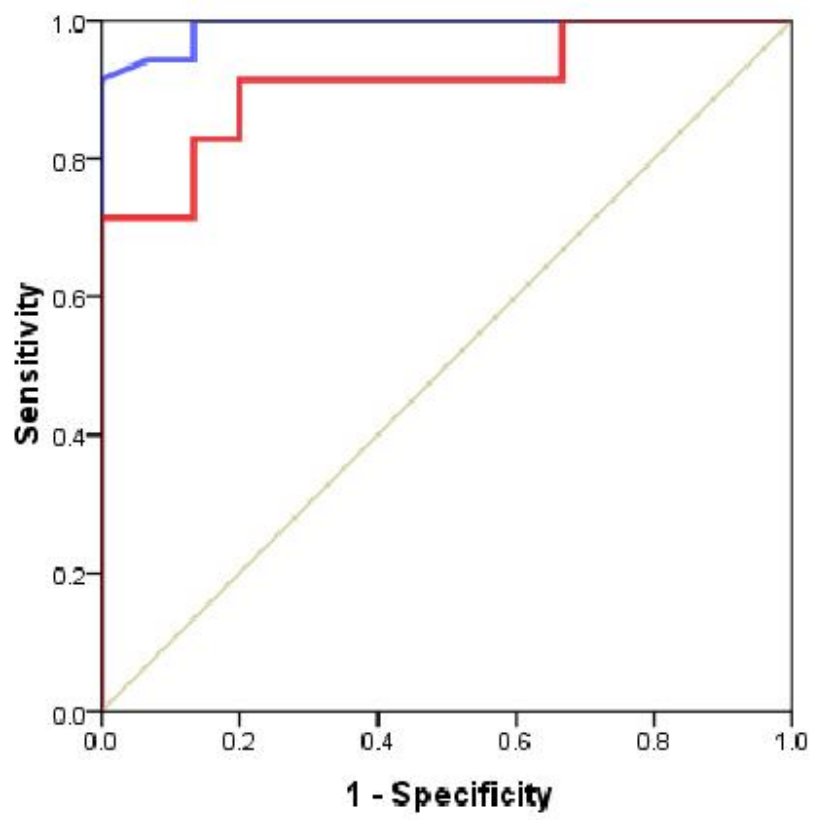

Source of the Curve

-micro RNA-21

- micro RNA-92

Reference Line

Fig. (5): ROC curve for the performance of miR-21

and miR-92 in CRC detection.

\section{DISCUSSION}

CRC is the third most prevalent cancer worldwide and is a leading cause of cancer-related mortality for both men and women [19]. In Egypt, CRC is one of the most common malignant neoplasms. It ranks the sixth most common cancer in both males and females [20,21]. The median age of CRC cases in Egypt is 48 and $51.5 y_{\mathrm{s}}$ for males and females, respectively [22].

In the present study, the age of the studied cases ranged between 19-74ys, with a mean of $50.6 \pm$ 15.1 ys. More than $60 \%$ were $>50 y$ s and $26 \%$ (more than $1 / 4$ of patients) were $\leq 40 \mathrm{ys}$ old. This comes in agreement with many Egyptian studies Gado et al. [22] found that the mean age of their assessed patients was 51ys and 44.8ys respectively and El Attar [23] and Sakr et al. [21] told that the mean age of their studied patients was 48ys and 51.2 ys respectively.

Males represented $56 \%$ of the studied cases in the present study. Rim et al. [24] and Murphy et al. [25] reported that men have more incidence of CRC than women, while El-Bolkainy et al. [26], Abotchie et al. [27] and Sakr et al. [21] told that CRC affects men and women almost equally

The percentage of the studied cases with CRC with a positive family history was $17 \%$. This comes in agreement with chan et al. [28] and Haggar \& Boushey [29] who told that the percentage of patients with CRC with a positive family history was up to $20 \%$. Fatemi et al. [30] reported a higher figure $(31.3 \%)$ in their studied Iranian patients. These data make adherence to CRC screening programs is mandatory in subjects with family history of CRC. Lieberman and his colleagues [31] told that screening should be offered to individuals with a family history of CRC earlier than for the average-risk population.

As regards the tumor site, colonic lesions (68.6\%) were encountered more than rectal $(25.7 \%)$ and rectosigmoid (5.7\%) ones. Yi et al. [32] reported similar finding of a lower incidence of rectal lesions (25.5-30.5\%) compared to colonic lesions. On the other hand, Kenawi et al. [33] and Soliman et al. [34] told that about half of their studied cases were rectal lesions, this disagreement may be attributed to the large number of their studied cases (400 cases) compared to the small number in our study.

Upon histopathological examination, most of the studied cases in the present study were adenocarcinoma (68.6\%). Mucinous and signet ring adenocarcinomas constituted around 25.7 and $5.7 \%$ respectively. This comes in agreement with Hamilton et al., (2010) ${ }^{49}$, Veruttipong et al. [35], Said et al. [36] and Sakr et al. [21].

Our results exhibited significantly higher mean serum miR-21 and miR-92a levels $(\mathrm{P}<0.001)$ in the studied cases compared to the control group 
(5.53 \pm 0.17 and $7.01 \pm 0.234 \neq 4.82 \pm 0.20$ and $6.56 \pm 0.20 \log \mathrm{RU}$, respectively). This was in agreement with, Wang et al. [37] who found that miR-21\& miRNA-92a levels were significantly higher in CRC cases and added that miR92a could distinguish CRC and advanced adenoma from normal controls, with a sensitivity of $>62 \%$ and specificity of $>84 \%$. No statistically significant association was found between serum miR-21 or miR-92a level and either age or sex. This comes in agreement with Guang-Hui and colleagues [38].

The mean value of serum miR-21 level showed a statistically significant positive relationship with the tumor size $(\mathrm{P}<0.001)$ and the mean values in small $(<2.5 \mathrm{~cm})$, medium and large $(>5 \mathrm{~cm}) \mathrm{CRC}$ lesions were 5.22, 5.51 and $5.61 \log \mathrm{RU}$, respectively. Also serum miR-21 level showed a statistically significant positive relationship with the pathological grade of CRC $(\mathrm{P}<0.05)$. The mean value of grade I was (5.27 $\log \mathrm{RU})$, grade II (5.51 $\log$ RU) and grade III (5.64 $\log$ RU). Higher mean values of serum miR-21 were found in higher CRC pathological grades. This comes in agreement with Toiyama et al. [39]. In the present study, the mean value of serum miR-21 level showed a statistically significant difference in different TNM stages $(\mathrm{P}>0.05)$ with a statistically significant positive correlation. The mean values in Tis-T2, T3 and T4 were 5.19, 5.53 and $5.59 \log \mathrm{RU}$, respectively. This comes in agreement with Schetter et al. [40] who found that higher expression levels of miR-21 were associated with more advanced clinical stages of CRC. The mean value of serum miR-21 level showed a statistically significant difference between different MAC Coller stages $(\mathrm{P}>0.05)$ in the present study with a statistically significant positive correlation. The mean values in A-B1, $\mathrm{B} 2$ and $\mathrm{B} 3$ were $5.19,5.55$ and $5.58 \log \mathrm{RU}$, respectively.

The mean value of serum miR-92a level showed a statistically significant positive correlation with tumor size $(\mathrm{P}<0.003)$. The mean values in small, medium and large CRC lesions were 6.75, 6.93 and $7.13 \log \mathrm{RU}$, respectively This comes in agreement with Zhou et al. [41] who reported that over expression of miR-92a is correlated with TNM stages and poor prognosis in CRC. Nami and his colleagues [42] told that a significant increase in miR-92a expression was more frequently observed in CRCs than in colorectal adenomas, and suggested that miR-92a could be a potential marker for discrimination between cancers and adenomas and a potential promoter for the phenotypic changes from adenoma into carcinoma. They added that miR-92a expression was related to advanced clinical stages and to the depth of invasion.

The mean value of serum miR-92a level showed a statistically significant positive correlation and different TNM stages $(\mathrm{P}>0.03)$. The mean values in Tis-T2, T3 and T4 were 6.67, 7.01 and $7.08 \mathrm{log}$ $\mathrm{RU}$, respectively .This comes in agreement Zhang et al. [43] who reported that expression of miR92a was associated with more advanced tumorlymph node-metastasis (TNM) stage $(\mathrm{P}=0.07)$.

When ROC curves were applied; serum miR-21 at a cutoff value of $\geq 5.25 \log \mathrm{RU}$ was $94.3 \%$ sensitive and $93.3 \%$ specific for diagnosis of $\mathrm{CRC}$ with an AUROC $=0.99$. Wang et al. [37] reported that serum miR-21 showed a sensitivity and specificity of $93 \%$ and $91 \%$, respectively. While, Ahmed et al. [44] and Schetter et al. [40] reported that serum miR-21 could detect CRC with $90 \%$ sensitivity and specificity. On the other hand, Guang-Hui et al. [38] reported that the sensitivity of serum miR-21 for detection CRC was only $65 \%$ and the specificity was $85 \%$.

In the present study, serum miR-21 level $\geq 5.61$ Log RU, could significantly discriminate cases of CRC lesions larger than $5 \mathrm{~cm}$ with $83.3 \%$ sensitivity and $82.4 \%$ specificity, with AUROC $=0.87$ as well as cases with high grade adenocarcinoma with $100 \%$ sensitivity and $63.3 \%$ specificity and AUROC $=0.85$. At a level $\geq 5.43$ $\log \mathrm{RU}, \mathrm{miR}-21$ could significantly detect cases of $\mathrm{T}$ stage $>2$ with $90 \%$ sensitivity and $100 \%$ specificity, with AUROC $=0.95$, and at a level $\geq 5.6 \log \mathrm{RU}$, it could significantly detect cases of MaCollerB3 with $68.4 \%$ sensitivity and $68.8 \%$ specificity, with AUROC $=0.71$.

Serum miR-92a at a cut off value of $\geq 6.75 \mathrm{Log}$ RU, was $91.4 \%$ sensitive and $80 \%$ specific for diagnosis of CRC with an AUROC $=0.91$. Other studies reported plasma miR-92a to have a sensitivity and specificity of $89 \%$ and $70 \%$ respectively at a cut off value of $\geq 6.75 \log R U$ in distinguishing CRC patients from healthy controls [43,44]. Huang et al. [14] also reported similar results (sensitivity: 84 , specificity: 71.4). In addition, many studies have found miR-92a expression to be associated with other diseases such as hepatocellular carcinoma, breast cancer, and even cardiovascular diseases, pointing to the low specificity of miR-92a. MiR-92a which has been studied extensively in plasma has also been 
observed to have higher expression levels in the stool of CRC patients [13]. However, the sensitivity and specificity of the miRNA 92a for detecting CRC was significantly lower in stool than in plasma[13].

The present study found that serum miR-92a at a cut off value $\geq 7.05 \log \mathrm{RU}$, could significantly detect cases of CRC lesions larger than $5 \mathrm{~cm}$ with $72.2 \%$ sensitivity and $70.6 \%$ specificity, with AUROC $=0.82$. This was in agreement with Guang et al. [38] who reported that, at a cut off $\geq 7.0$, AUROC value of $0.722(95 \% \mathrm{CI}=0.633$ 0.811 ) with a sensitivity of $70 \%$ and specificity of $70 \%$. At level $\geq 7.1 \log \mathrm{RU}$, miR-92a could significantly detect cases of MaC CollerB3 with $63.2 \%$ sensitivity and $81.8 \%$ specificity, with AUROC $=0.76$.This was in agreement with Guang et al. [38] who told that serum levels of miR -92a were potential biomarkers for CRC, and at the cut-off value of $\geq 7.1$, the sensitivity was $65.5 \%$ and the specificity was $82.5 \%$ with an AUROC $=0.786$.

At level $\geq 6.96 \log$ RU, miR-92a could significantly detect cases of $\mathrm{T}$ stage $>2$ with $77.4 \%$ sensitivity and $100 \%$ specificity, with AUROC $=0.9$ This was in agreement with Guang et al. [38].

When serum miR-21 ( $\geq 5.25 \log \mathrm{RU})$ and miR92a ( $\geq 6.75 \log R U)$ were combined together, the sensitivity for detection of CRC was increased to $97.1 \%$ and the specificity was $93.3 \%$.

In conclusion, serum miR-21 and miR-92a expression levels represent a sensitive and specific tool for detection of CRC with higher accuracy of miR-21.

Funding: None.

\section{Conflicts of interest: None.}

\section{Ethical approval: Approved}

\section{REFERENCES}

1- Croner RS, Geppert CI, Bader FG, Nitsche U, Spath C, Rosenberg R, et al. Molecular staging of lymph node-negative colon carcinomas by onestep nucleic acid amplification (OSNA) results in upstaging of a quarter of patients in a prospective, European, multicentre study. Br J Cancer, 2014. 110(10): p. 2544-50.

2- Gellad ZF and Provenzale D, Colorectal cancer: national and international perspective on the burden of disease and public health impact. Gastroenterology, 2010. 138(6): p. 2177-90.
3- Siegel R, Naishadham D, and Jemal A, Cancer statistics, 2013. CA Cancer J Clin, 2013. 63(1): p. 11-30.

4- Jemal A, Bray F, Center MM, Ferlay J, Ward E, and Forman D, Global cancer statistics. $C A$ Cancer J Clin, 2011. 61(2): p. 69-90.

5- Wang Z, Lu Y, Zhang X, Ren X, Wang Y, Li Z, et al., Serum microRNA is a promising biomarker for osteogenesis imperfecta. Intractable Rare Dis Res, 2012. 1(2): p. 81-5.

6- He L, He X, Lim LP, De Stanchina E, Xuan Z, Liang Y, et al., A microRNA component of the p53 tumour suppressor network. Nature, 2007. 447(7148): p. 1130-4.

7- Agostini M, Pucciarelli S, Calore F, Bedin C, Enzo M, and Nitti D, miRNAs in colon and rectal cancer: A consensus for their true clinical value. Clin Chim Acta, 2007. 411(17-18): p. 1181-6.

8- Chin LJ and Slack FJ, A truth serum for cancer-microRNAs have major potential as cancer biomarkers. Cell Res, 2008. 18(10): p. 983-4.

9- Wang CJ, Zhou ZG, Wang L, Yang L, Zhou B, $\mathrm{Gu}$ J, et al., Clinicopathological significance of microRNA-31, -143 and -145 expression in colorectal cancer. Dis Markers, 2009. 26(1): p. 27-34

10- Asangani IA, Rasheed SA, Nikolova DA, Leupold JH, Colburn NH, Post S, et al., Micro RNA-21 (miR-21) post-transcriptionally downregulates tumor suppressor Pdcd4 and stimulates invasion, intravasation and metastasis in colorectal cancer. Oncogene, 2008. 27(15): p. 2128-36.

11- Kanaan Z, Rai SN, Eichenberger MR, Roberts H, Keskey B, Pan J, et al., Plasma miR-21: a potential diagnostic marker of colorectal cancer. Ann Surg, 2012. 256(3): p. 544-51.

12- Slaby O, Svoboda M, Fabian P, Smerdova T, Knoflickova D, Bednarikova M, et al., Altered expression of miR-21, miR-31, miR-143 and miR-145 is related to clinicopathologic features of colorectal cancer. Oncology, 2007. 72(5-6): p. 397-402.

13- Wu CW, Ng SS, Dong YJ, Ng SC, Leung WW, Lee CW, et al., Detection of miR-92a and miR-21 in stool samples as potential screening biomarkers for colorectal cancer and polyps. Gut, 2012. 61(5): p. 739-45.

14- Huang Z, Huang D, Ni S, Peng Z, Sheng W, and $\mathrm{Du} \mathrm{X}$, Plasma microRNAs are promising novel biomarkers for early detection of colorectal cancer. Int J Cancer, 2010. 127(1): p. 118-26.

15- Sequences.Txt H.U.-U.E.B.P.M. 
16- Greenberg R, Daniels R, Flanders W, Eley J, and Boring J, Diagnostic testing In Medical epidemiology. McGraw-Hill, New York, NY, 1996. 3rd edition: p. 77-89.

17- Siegel R, Desantis C, and Jemal A, Colorectal cancer statistics, 2014. CA Cancer J Clin, 2014. 64(2): p. 104-17.

18- Kothari CR, Research Methodology: Methods and Techniques. New Age International Publishers, New Delhi, 2004. 2nd Edition.

19- Zeeneldin A, Saber MM, El-Din I, and Frag S, Colorectal carcinoma in gharbiah district, Egypt: Comparison between the elderly and non-elderly. Journal of Solid Tumor, 2012. 2(3): p. 13-23.

20- Locker GY, Hamilton S, Harris J, Jessup JM, Kemeny N, Macdonald JS, et al., ASCO 2006 update of recommendations for the use of tumor markers in gastrointestinal cancer. J Clin Oncol, 2006. 24(33): p. 5313-27.

21- Sakr SA, Badrah GA, and Sheir RA, Histological and histochemical alterations in liver of chronic hepatitis C patients with Helicobacter pylori infection. Biomed Pharmacother, 2013. 67(5): p. 367-74.

22- Gado A, Ebeid B, and A A, Colorectal cancer in Egypt is commoner in young people: Is this cause for alarm?. Alexandria Journal of Medicine; 2014. 50(3): p. 197-201.

23- El-Attar I, Colo-Rectal Cancer: Magnitude of the Problem. Annual Cancer Conference of the Egyptian Cancer Society, Danish Cancer Society \& Aarhus University Hospital, 2005. Cairo, 9-11 February.

24- Rim SH, Seeff L, Ahmed F, King JB, and Coughlin SS, Colorectal cancer incidence in the United States, 1999-2004 : an updated analysis of data from the National Program of Cancer Registries and the Surveillance, Epidemiology, and End Results Program. Cancer, 2009. 115(9): p. 1967-76.

25- Murphy G, Devesa SS, Cross AJ, Inskip PD, Mcglynn KA, and Cook MB, Sex disparities in colorectal cancer incidence by anatomic subsite, race and age. Int J Cancer, 2011. 128(7): p. 166875.

26- El-Bolkainy TN, Sakr MA, Nouh AA, and El-Din NH, A comparative study of rectal and colonic carcinoma: demographic, pathologic and TNM staging analysis. J Egypt Natl Canc Inst, 2006. 18(3): p. 258-63.

27- Abotchie PN, Vernon SW and Du XL, Gender differences in colorectal cancer incidence in the United States, 1975-2006. J Womens Health (Larchmt), 2012. 21(4): p. 393-400.
28- Chan JA, Meyerhardt JA, Niedzwiecki D, Hollis D, Saltz LB, Mayer RJ, et al., Association of family history with cancer recurrence and survival among patients with stage III colon cancer. JAMA, 2008. 299(21): p. 2515-23.

29- Haggar FA and Boushey RP, Colorectal cancer epidemiology: incidence, mortality, survival, and risk factors. Clin Colon Rectal Surg, 2009. 22(4): p. 191-7.

30- Fatemi SR, Malek FN, Shivarani S, Vahedi M, Almasi S, Maserat E, et al., Prevalence of colorectal cancer in relatives of Iranian patients diagnosed with colorectal cancer. Asian Pac J Cancer Prev, 2010. 11(1): p. 91-3.

31- Lieberman DA, Rex DK, Winawer SJ, Giardiello FM, Johnson DA, and Levin TR, Guidelines for colonoscopy surveillance after screening and polypectomy: a consensus update by the US Multi-Society Task Force on Colorectal Cancer. Gastroenterology, 2012. 143(3): p. 844-857.

32- Yi M, Xu J, Liu P, Chang GJ, Du XL, Hu CY, et al., Comparative analysis of lifestyle factors, screening test use, and clinicopathologic features in association with survival among Asian Americans with colorectal cancer. Br J Cancer, 2013. 108(7): p. 1508-14.

33- Kenawi Mm, A E-S, and N EB, Relative frequency of gastrointestinal malignancies at NCI during 14 years (1976-1989). Med J Cairo Uni, 1990. 58: p. 35-41.

34- Soliman AS, Bondy ML, Hamilton SR, and Levin $\mathrm{B}$, Colon cancer in young Egyptian patients. Am J Gastroenterol, 1999. 94(4): p. 1114.

35- Veruttipong D, Soliman AS, Gilbert SF, Blachley TS, Hablas A, Ramadan M, et al., Age distribution, polyps and rectal cancer in the Egyptian population-based cancer registry. World J Gastroenterol, 2012. 18(30): p. 3997-4003.

36- Said M, Saber M, M Khairy, El-Hendawy A, Khalf O, Abdelbary M, et al., Lack of estrogen receptors expression in malignant and premalignant colorectal lesions in Egyptian patients. Open Journal of Gastroenterology, 2013. 2(2).

37- Wang J, Huang SK, Zhao M, Yang M, Zhong JL, $\mathrm{Gu} \mathrm{YY}$, et al., Identification of a circulating microRNA signature for colorectal cancer detection. PLoS One, 2014. 9(4): p. e87451.

38- Liu GH, Zhou ZG, Chen R, Wang MJ, Zhou B, Li Y, et al., Serum miR-21 and miR-92a as biomarkers in the diagnosis and prognosis of colorectal cancer. Tumour Biol, 2013. 34(4): p. 2175-81. 
39- Toiyama Y, Takahashi M, Hur K, Nagasaka T, Tanaka K, Inoue Y, et al., Serum miR-21 as a diagnostic and prognostic biomarker in colorectal cancer. J Natl Cancer Inst, 2013. 105(12): p. 84959.

40- Schetter AJ, Leung SY, Sohn JJ, Zanetti KA, Bowman ED, Yanaihara N, et al., MicroRNA expression profiles associated with prognosis and therapeutic outcome in colon adenocarcinoma. JAMA, 2008. 299(4): p. 425-36.

41- Zhou T, Zhang G, Liu Z, Xia S, and Tian H, Overexpression of miR-92a correlates with tumor metastasis and poor prognosis in patients with colorectal cancer. Int J Colorectal Dis, 2012. 28(1): p. $19-24$
42- Yamada N, Nakagawa Y, Tsujimura N, Kumazaki M, Noguchi S, Mori T, et al., Role of Intracellular and Extracellular MicroRNA-92a in Colorectal Cancer. Transl Oncol, 2013. 6(4): p. 482-92.

43- Zhang GJ, Zhou T, Liu ZL, Tian HP, and Xia SS, Plasma miR-200c and miR-18a as potential biomarkers for the detection of colorectal carcinoma. Mol Clin Oncol, 2013. 1(2): p. 379384.

44- Ahmed FE, Amed NC, Vos PW, Bonnerup C, Atkins JN, Casey M, et al., Diagnostic micro RNA markers to screen for sporadic human colon cancer in blood. Cancer Genomics Proteomics, 2012. 9(4): p. 179-92. 